\title{
Parrondo's Paradox: Gambling games from noise induced transport - a new study
}

\author{
Abhijit Kar Gupta ${ }^{1, a, *}$, Sourabh Banerjee ${ }^{2, b}$ \\ ${ }^{1}$ Department of Physics (UG \& PG), Panskura Banamali College (autonomous), Panskura R.S., \\ East Midnapore, Pin: 721 152, WB, India \\ ${ }^{2}$ Department of Physics, Government General Degree College at Kushmandi, P.O. Kalikamora, \\ South Dinajpur, Pin: 733 121, WB, India \\ a.kg.abhi@gmail.com, b.bandosou@gmail.com \\ *corresponding author
}

Keywords: Paradox, Ratchet, Brownian, Game, Gamble, Gambling, probabilistic game, coin tossing, Noise, Molecular motor

Abstract: Noise is known to disrupt a preferred act or motion. Yet, noise and broken symmetry in asymmetric potential, together, can make a directed motion possible, in fact essential in some cases. In Biology, in order to understand the motions of molecular motors in cells ratchets are imagined as useful models. Besides, there are optical ratchets and quantum ratchets considered in order to understand corresponding transport phenomena in physics. The discretized actions of ratchets can be well understood in terms of probabilistic gambling games which are of immense interest in Control theory - a powerful tool in physics, engineering, economics, biology and social sciences.

In this article, we deal with Parrondo's paradox which is about a paradoxical game and gambling. Imagine two kinds of probability dependent games A and B, mediated by coin tossing. Each of the games, when played separately and repeatedly, results in losing which means the average wealth keeps on decreasing. The paradox appears when the games are played together in random or periodic sequences; the combination of two losing games results into a winning game! While the counterintuitive result is interesting in itself, the model can very well be thought of a discretized version of Brownian flashing ratchets which are employed to understand noise induced order. In our study, we examine various random combinations of losing probabilistic games in order to understand how and how far the losing combinations result in winning. Further, we devise an alternative model to study the similar paradoxical game and examine the idea of paradox in it. The work is done by computer simulations. Analytical calculations to support this work, is currently under progress.

\section{Introduction: Parrondo’s Paradox Game and interpretation}

A gambling or a probabilistic game can be depicted by coin tossing. In this Parrondo's paradox game, gambling is done with two biased coins. It is easy to understand that when we play with a 
biased coin and we bet with some capital, we are either destined to lose or win for sure. So in this case, the game is not fair in a sense that our average capital does not remain constant as we keep playing such an unfair game repeatedly. Now let us imagine two differently designed losing games; the games are unfair and we are going to lose (as we play repeatedly) when played separately. Now, the question is, if we play the two kinds of games together, what we will happen? Do we lose or can we win? The surprising answer is that we can win! In other words, two losing games can be combined to form a winning game. This is counterintuitive to think and appears as a paradox. This game is known as Parrondo's paradox as that was first devised and studied by a physicist, Juan M.R. Parrondo [1].

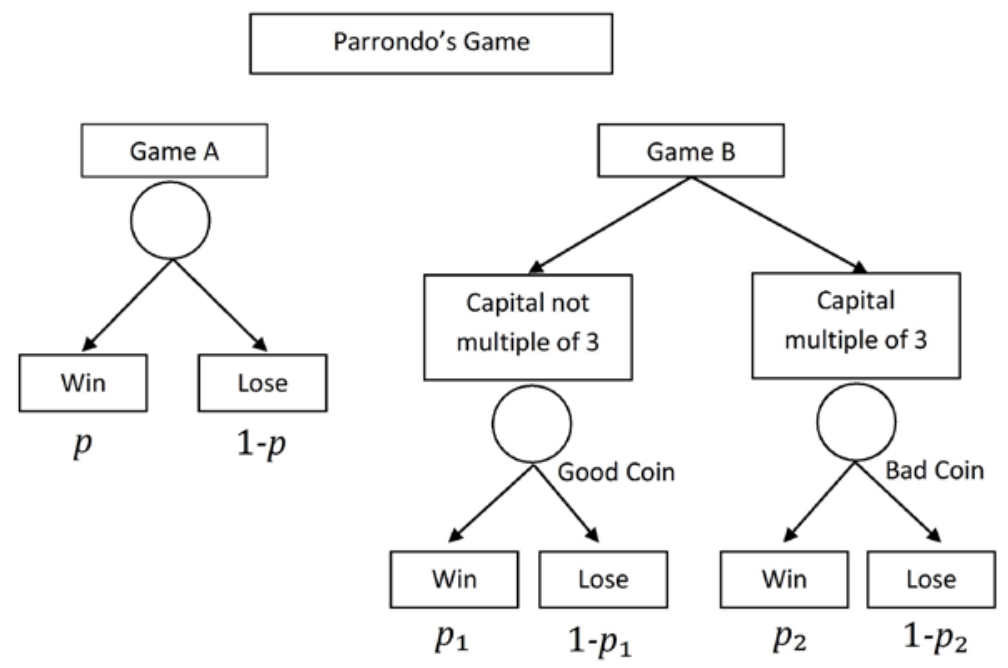

Figure 1: Demonstration of Parrondo's Paradox Game: In the original game, and for our consideration here in this work, winning probabilities of different coins, $p=0.5-0.005$, $p_{1}=0.75-0.005$ and $p_{2}=0.1-0.005$.

In the above block diagram [Figure 1], we demonstrate the original Parrondo's Paradox scenario. Let us consider two simple coin tossing games A and B. Each time, if we win, our capital is increased by 1 unit and loosing makes our capital to decrease by 1 unit. Game A is played with a coin which is a slightly biased towards losing: the probability of winning being $p=0.5-\varepsilon$, where $\varepsilon=0.005$ as considered as a biasing parameter. Game B is somewhat more complex. If the capital is not modulo 3 (that is not divisible by 3), then one has to play with a 'good coin', biased coin towards winning: with the probability of winning being $p_{1}=0.75-\varepsilon$. When the capital is a multiple of 3 , one has to play with 'bad coin', heavily biased coin towards losing and in this case the probability of winning is $p_{2}=0.1-\varepsilon$.

As mentioned, if one plays game A or game B separately and repeatedly, each of them is a losing game. A simple computer program can be written to plot the cumulative mean capital against number of iterations. For game A, it is quite easy to understand. But for game B, one needs to go through some heuristic arguments based on Markov chain process to arrive at the conclusion (The arguments and necessary mathematics are provided in [2], a beautiful review by Parrondo et al.) The outcomes of game A and game B, played separately and when played together, had been demonstrated through mathematical considerations and numerical simulations whereas some variants of the paradoxical game are also studied in the literature. Out of the models, that are considered, two main ideas consist of how and when game A and B are combined. In the original 
game, the good coin or bad coin in game B depended on the value of the capital, so it is called capital dependent game. In other kind of variation, coins in game B will be chosen while looking at the wining or losing history of the game, and not on the capital. This latter version is called history dependent game.

Even though Parrondo's Paradox is a simple toy model to lead us to investigate the counterintuitive situation, it opens up a new way to analyse and understand the functioning of Brownian ratchets and flashing Brownian ratchets in particular [6]. Brownian ratchets are connected with Brownian motors [7] which are essentially some asymmetric potential dependent phenomena where directed move in presence of noise or fluctuations is possible. It is important to understand how tiny little protein based molecular motors move in the cell environment with intracellular thermal noise as well as under macroscopic environmental noise. There are plenty of applications and the interest lies in modeling in biology, physics, economics and other social sciences. A flashing Brownian ratchet is where the asymmetric potential is switched on and off in some fashion.

A simple flashing Brownian ratchet [Figure 2] consists of a series of asymmetric potential on a line. If the particles are trapped in one minimum, they can not move due to asymmetric potential walls. In the absence of it, particles can diffuse freely on either sides, right or left. In this present scenario, in the absence of asymmetric potential, there is a force towards left with the help of a slanting potential. But when the ratchet potential is on again, many of the particles are forced to move towards right than on the left even with the presence of the small applied force towards left. Particles can move on the left when the ratchet potential is switched off or on. But it can be demonstrated that when the ratchet potential is made off and on randomly or periodically, a net rightward motion is possible even in the presence of the force toward left. This is called flashing ratchet and the two states (off and on of ratchet potential) can be thought of as two games A and B with negative outcome.

Noise or fluctuation is a considerable nightmare in the fields of physics, biology or electrical engineering. But noise induced movement or stochastic resonance has lately gained importance in such fields. If we consider fluctuation as a probability then we can think of probabilistic games or gambling in order to understand the interplay of noises or fluctuations in the systems. Parrondo's paradox is thus an approach where we can identify the roles of negativities that can bring out a positive outcome.

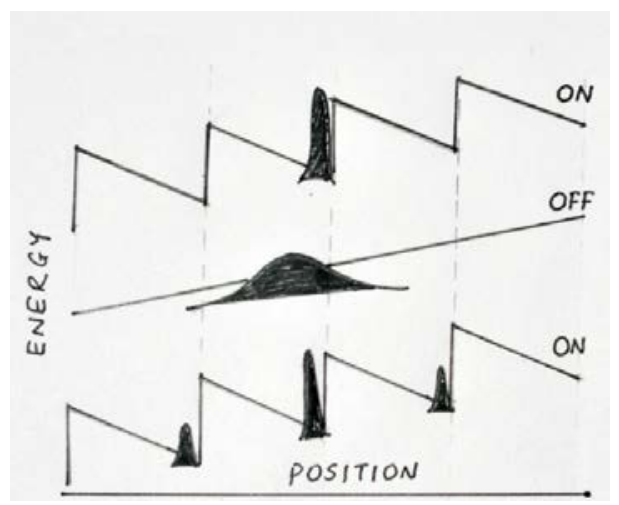

Figure 2: Demonstration of Flashing ratchet 


\section{Some Numerical Results for understanding: Capital dependent game}

As discussed above, if we take a combination of game $\mathrm{A}$ and $\mathrm{B}$, such that we alternate the games like AABBAABB....or in some other sequence or even in a random fashion, it is seen that the resulting capital goes on increasing with the number of turns they are played. In figure 3 , we illustrate with numerical results for different situations where the average capital is plotted with the number of times the games played.

In order to understand some essence of the paradox, we can try with computer simulations and check. It may be said that the number of times the bad coin is played (in game B) can be controlled through the capital. Since the association of game A makes it winning, the effect must be that the number of times the bad coin is used must have been reduced when game A is played along with. This has been found to be true as checked by us numerically.

Original Parrondo's Paradox

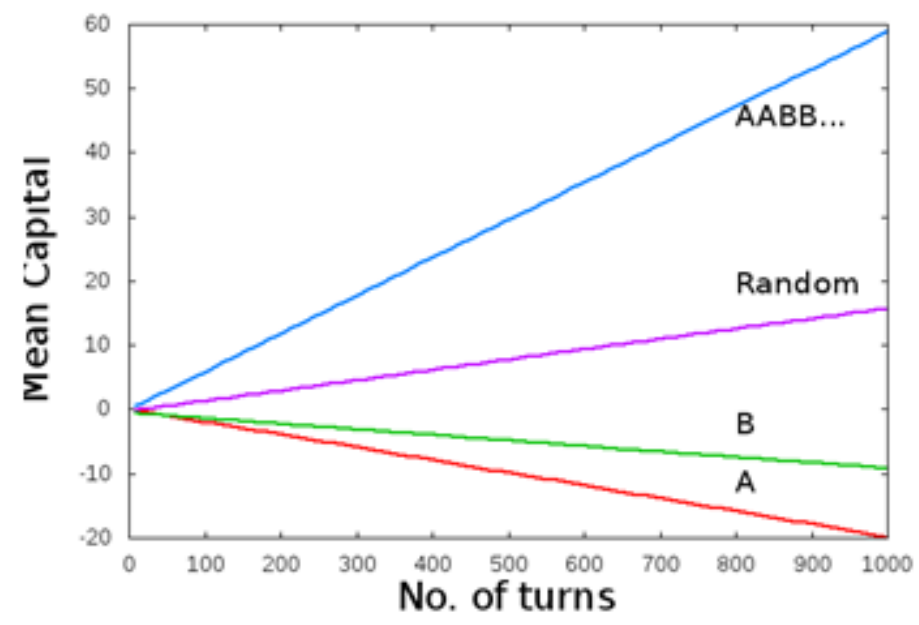

Figure 3: Capital dependent game. The mean capitals against number of turns are plotted for four different cases. For games A or B played separately, it is clearly seen that the mean capital is decreasing monotonically and for the combined games (AABBAABB sequence or with random A and $\mathrm{B})$, that is increasing monotonically.

The effect of playing game A can be thought of that the capital in such a way that the capital turns less likely to be a multiple of 3 . The connection (between two games) and control are through the value of the capital (capital dependent game [2]). Other variations, such as capital independent (or history dependent) game [3] and detailed numerical studies of these models are reported in literature [4].

\section{New Results: Random combinations of two losing games}

We have studied the Parrondo's paradox game, as outlined above, in more detail and tried to figure out how far the combination of two losing games $\mathrm{A}$ and $\mathrm{B}$ can give rise to winning situation. In this combined game, where the sole connection between games A and B is through the capital, the outcome of the game (win or loss) would depend on the relative appearance of game A with respect to game $B$. With some periodic combinations of A and B (for example, AABBAABB...), we have checked the resulting paradoxical behaviour, that is the resulting game is winning which means the mean capital of a player increases with the number of turns played. However, our idea has been to examine the combined game where one plays $\mathrm{A}$ and $\mathrm{B}$ in random order. 
The questions to ask, will the resulting game be always winning with any proportion of $\mathrm{A}$ or $\mathrm{B}$ played? To look for an answer, we simulated the combined games where game A is played with probability $p$ and game $\mathrm{B}$ with $(1-p)$ in random order. For each value of $p[0,1]$, the simulation is done to check the resulting mean capital for reasonably long time steps to make sure of the trend, i.e., if the combined game is winning or losing. But interestingly, it is observed that with any arbitrary amount of randomness, the combined game is not always winning. For a certain range of values of $p$, the game appears to be winning and for higher or lower values than that, the combined game is losing which means the paradox does not occur. The numerical result is displayed below. The preliminary understanding is that too much or too little presence of game A influences the capital in such a way that the triggering of the good coin in game B turns less and less. Game A pushes the value of capital towards or away from the multiple of 3 , which the game $\mathrm{B}$ cannot do alone. The mechanism in B changes the capital toward multiple of 3 more likely (mathematical argument is given in [2]) and that is the bad coin is played more often when game B is played only. These pathological scenarios are checked with detailed numerical investigations [Figure 4].

Parrondo's Paradox: effect of randomness

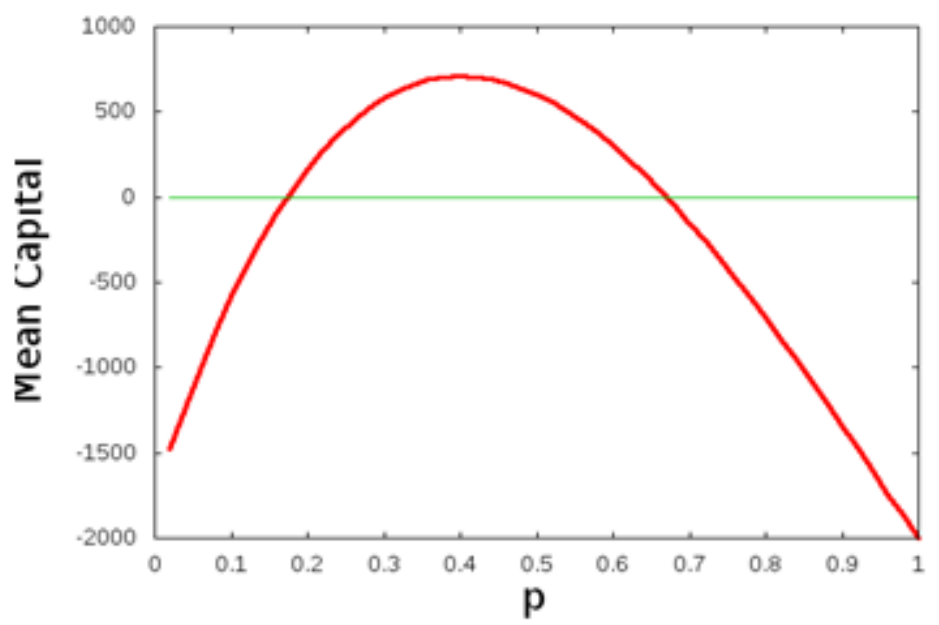

Figure 4: Variation of mean capital against the probability $(p)$ of appearance of game A.

\section{A New History dependent Game}

It is apparent that in the above described capital dependent game, the connection between two games A and B is the capital. The condition applied over capital (multiple of 3 or not) is what responsible for switching from one coin to other (good or bad) and hence affects the net outcome. Now the idea is, if the similar switching can be done by some other means, presumably the similar paradox might happen. One such attempt has been done through the history of the games played. Such a history dependent game [5] was devised where game A was kept intact and the game B to be played with 4 different biased coins depending on the history of winning or losing in the previous two turns. However, instead of such a complicated history dependent game, we propose a simpler version of such a history dependent game. In this new game, we obtain the paradox while playing the game $\mathrm{B}$ with two coins only (as is done in original capital dependent game).

In the new history dependent game (as demonstrated in the following table), we keep the game A the same [Win with $0.5-\epsilon$ and loss with $0.5+\epsilon$ ]. Also, the game B is played with two coins: good coin and bad coin, with same probabilities as before (Good Coin: winning probability $0.9-\epsilon$, Bad Coin: winning probability $0.4-\epsilon$ ). In our newly devised history dependent game, we play a 
trick over when to play with the good coin or the bad coin. The history dependence is described in the table.

Table 1: The New History Dependent Game. The possibilities with the two coins used.

\begin{tabular}{|l|l|l|}
\hline Step $t-2$ & Step $t-1$ & $\begin{array}{l}\text { Coin used } \\
\text { in Game B }\end{array}$ \\
\hline Lose & Lose & $\begin{array}{l}\text { Good coin } \\
{[\text { Win with }} \\
p=0.9-\epsilon]\end{array}$ \\
\hline Lose & Win & $\begin{array}{l}\text { Bad Coin } \\
{[p=0.4-\epsilon]}\end{array}$ \\
\hline Win & Lose & Bad Coin \\
\hline Win & Win & Bad Coin \\
\hline
\end{tabular}

Now, as before, like that of capital dependent game, when the games A and B are played separately, they are losing games in the sense that the average capital decreases with the number of turns. However, with a combination of them, for example, AABAAB... (where game A is played consecutively twice and then game B and so on...), the result is winning. A computer simulation is done and that is demonstrated in figure 5. [For mean capital, numerical results are obtained with 10,000 configurations in each case.]

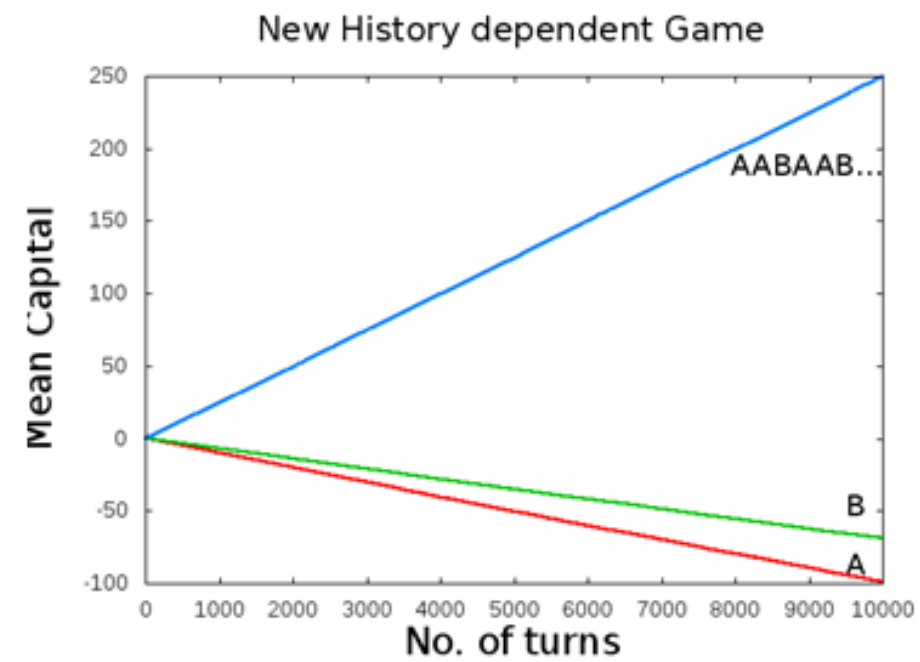

Figure 5: The evolution of mean capital against the number of turns when the games are individually played (A or B) and the case for a periodic combination of game A and game $\mathrm{B}$.

The essence of Parrondo's paradox is that the combination of two losing games can give rise to a winning game. We have checked this with the original capital dependent game and then discovered that not all combinations can produce a winning game. For random combinations of A and B, we observed that the winning or losing of the combined game would depend on the probability of the appearance of game A (or B) in the seqyence. We checked the similar situation for this new model too and the computer simulation shows a similar scenario [Figure 6]. The mean capital (obtained after a certain number of turns) is first negative with lower probability, then positive for some intermediate values of probability $(p)$, and finally it turns out negative again. This means, the 
combined game is losing for lower and higher probabilities (of the appearance of A or the number of times A game is played) and winning for a certain range of probability of appearance of game A.

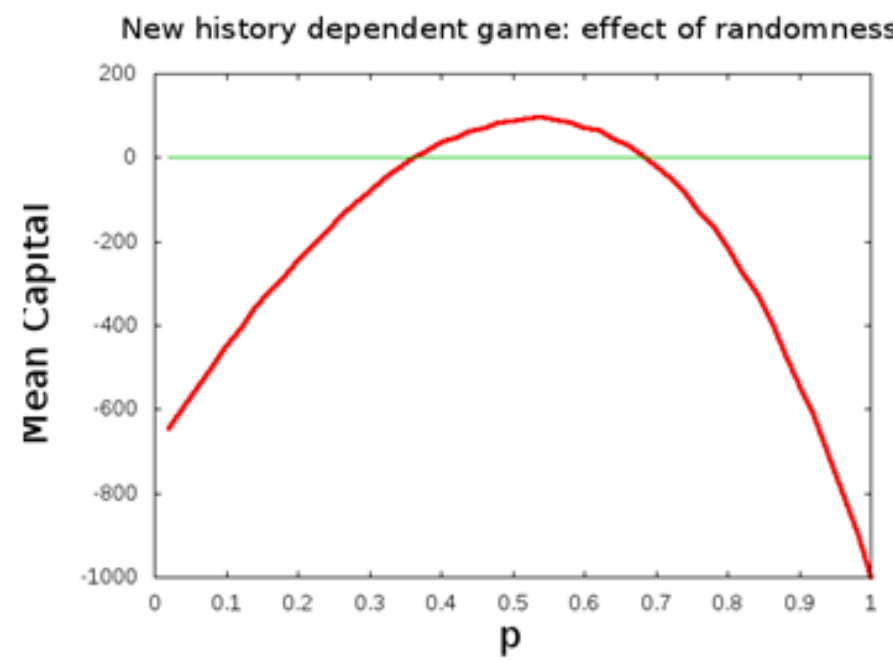

Figure 6: The variation of mean capital against the probability $(p)$ of the game A that appears in the New History Dependent Game.

\section{Conclusions}

There are a plenty of examples from physics to biology and in social sciences where the stochastic thermal fluctuations or other kinds of noise actually help achieving positive movements. It is in this context, the Brownian ratchets and the kind of prototype paradoxical games may be explored in detail.

We have studied original Parrondo's paradox where two losing probabilistic games (A and B) are combined to make a winning game. The two games can be combined in some ordered sequence or they can be selected randomly. However, our study reveals that the random selection of a game (A or B) plays an important role; the combined game can be winning or losing depending on the strength of randomness i.e., the probability $(p)$ of appearance of a game (say, A). Two variations of Parrondo's paradox game have been studied: capital dependent and history dependent ones. We have devised a new history dependent game, a simpler version compared to the usual history dependent game studied so far. In this new model too, we have examined the role of relative appearance of a particular component game (say, A) with respect to the other and we obtain the similar behaviour of winning or losing with respect to the strength of randomness. As a whole, our interest has been to study how and how far the paradox of winning from two losing games stands.

\section{References}

[1] Parrondo J. M.R. (1996), How to cheat a bad mathematician, EEC HC\&M Network on Complexity and Chaos (\#ERBCHRX-CT940546), ISI, Torino, Italy (unpublished).

[2] Parrondo J. M.R., Dinis L. (2004), Brownian motion and gambling: from ratchets to paradoxical games, Contemporary Physics, 45 (2), 147-157; arXiv:1410.0485v1 [physics.soc-ph].

[3] Dinis L., Parrondo J.M.R. (2003), Optimal strategies in collective Parrondo games, Europhys. Lett., 63, $319-325$.

[4] Shu Jian-Jun, Wang Qi-Wen (2014), Beyond Parrondo's Paradox, Sci. Rep., 4, 4244; DOI:10.1038/srep04244.

[5]Parrondo J.M.R., Harmer George P., and Abbott D. (2000), New Paradoxical Games Based on Brownian Ratchets, Phys. Rev. Lett., 85, 5226.

[6] Harmer G.P. and D. Abbott (1999), Game Theory - Losing strategies can win by Parrondo's paradox, Nature (London) 402, 846.

[7] Astumian R.D., Hänggi P., Brownian Motors (2002), Phys. Today, Nov., 33-39. 